\title{
Energy-Momentum Tensor and Parameters in Cosmological Model
}

\author{
Ying-Qiu $\mathrm{Gu}^{*}$ \\ School of Mathematical Science, Fudan University, Shanghai 200433, China
}

(Dated: 24th February 2020)

\begin{abstract}
In cosmology, the cosmic curvature $K$ and the cosmological constant $\Lambda$ are two most important parameters, whose values have strong influence on the behavior of the universe. By analyzing the energy-momentum tensor and equations of state of ideal gas, scalar, spinor and vector potential in detail, we find that the total mass density of all matter is always positive, and the initial total pressure is negative. Under these conditions, by qualitatively analyzing the global behavior of the dynamical equation of cosmological model, we get the following results: (i) $K=1$, namely, the global spatial structure of the universe should be a 3 -dimensional sphere $S^{3}$. (ii) $0 \leq \Lambda<10^{-24} \mathrm{ly}^{-2}$, the cosmological constant should be zero or an infinitesimal. (iii) $a(t)>0$, the initial singularity of the universe is unreachable, and the evolution of universe should be cyclic in time. This means that the initial Big Bang is impossible at all. Since the matter components considered are quite complete and the proof is very elementary and strict, these logical conclusions should be quite reliable. Obviously, these conclusions will be much helpful to correct some popular misconceptions and bring great convenience to further research other problems in cosmology such as property of dark matter and dark energy.
\end{abstract}

Keywords: Cosmological model; Energy-momentum tensor; Equation of state; Cosmic curvature; Cosmological constant; Negative pressure; Dynamic analysis.

PACS numbers: $98.80 . J k, 98.80 . \mathrm{Bp}, 98.80 . \mathrm{Cq}, 95.36 .+\mathrm{x}$

\section{Contents}

*Electronic address: yqgu@fudan.edu.cn 
A. Basic Equations in Cosmology

\section{INTRODUCTION}

In cosmology, we have two important constants to be determined. They are cosmic curvature $K$ and cosmological constant $\Lambda$. Some characteristic parameters of the universe, such as the age $\mathcal{T}$, Hubble constant $H_{0}$, total mass density $\Omega_{\text {tot }}$, etc., have been measured to high accuracy $\mathbb{1}$, 2, 3, 4, 5, 6]. To determine the cosmic curvature $K$, the usual approach is to transform the Friedmann equation into an algebraic equation $\Omega_{K} \equiv K \dot{a}^{-2}=\Omega_{\text {tot }}-1$. In theory, $K=0, \pm 1$ can be judged by contrasting observational data $\Omega_{\text {tot }}>1,=1$ or $<1$. From the observations we have $\Omega_{K}=-0.0020 \pm 0.0047$, which is very close to the case of flat space. Considering measurement errors, it is hard to determine what type the space is. In fact, no matter what the case of spatial curvature is, for a young universe, it is easy to calculate that, we always have $\Omega_{\text {tot }} \approx 1$. So this criterion is rather ambiguous.

Cosmological constant $\Lambda$ has a dramatic history. Since Einstein introduced $\Lambda$ in 1917 to get a static and closed universe, the debate over whether $\Lambda$ is zero or not has been repeated many times [7]-9]. Now dark matter and dark energy are attracting the attention of scientists around the world, becoming the hottest topic, challenging traditional standard models of particle and cosmology. The usual description of dark matter and dark energy is using the equation of state $P=w \rho$ and $w=w(a)$ or $w=w(z)$, and many specific models were obtained by fitting the observed data[7]-[14]. However, the problem is far from being solved[15].

In [12, by introducing the potential function $V(a)$, the Friedmann equations with some known dark energy models are converted into Hamiltonian dynamics, and the evolving trajectory is analyzed to explain the accelerating expansion of the universe. The literatures [16]-[22] provide some similar discussion on specific gravitational sources. The nonlinear scalar fields were discussed in 
reference [16, 17, 18] to obtain the cyclic universe model. In [19], a set of precise cyclic solutions with ordinary dust and radiation are obtained, and in [20], the exact solutions of ghost and electromagnetic fields are derived. The quantized nonlinear spinor fields and trajectories are calculated and a cyclic solution $a(t)$ is solved in [21, 22]. In [23, 24], the authors use $\left(\Omega_{K}, a\right)$ phase plane to discuss the dynamical behavior of the universe, and conclude that a cyclical universe is reasonable from a dynamical systems perspective, and requires in addition to standard cosmological assumptions, only two conditions: (i) the spatial sections must have positive spatial curvature $(K=+1)$, and (ii) the late time effective cosmological "constant" must decay fast enough as a function of the scale factor. Both of these conditions are consistent with all current observations to date. In 2008, M. Novello and S.E. Perez Bergliaffa reviewed the general features of nonsingular universes and cyclic universes, discussed the mechanisms behind the bounce, and analyzed examples of solutions that implement these mechanisms[25].

A recent paper published in 'Nature Astronomy' points out that our universe may be not flat [26], but rather more like a giant balloon that is closed and curved. After analyzing the Planck Legacy 2018 release, the authors found enhanced gravitational lens amplitude in the power spectrum of the cosmic microwave background(CMB), which is different from the data of the standard $\Lambda \mathrm{CDM}$ model. The main task of the Planck satellite is to detect the tiny fluctuation of the CMB temperature. The study of the fluctuation of the CMB temperature is the key to uncover the relevant cosmological models and parameters. This information defines the expansion, composition and origin of the cosmic large-scale structure. The authors use 'closed universe' to explain this anomalous effect. The spectra are now more inclined to a positive curvature greater than $99 \%$ confidence level. The positive curvature can explain the anomalous amplitude of the gravitational lens.

\section{ENERGY-MOMENTUM TENSOR OF MATTER}

In order to understand the mystery and dynamically behavior of the universe, Energymomentum tensor(EMT) and equation of state(EOS) of all kinds of matter are crucial factors. In this paper, we establish EMT and EOS of some elementary components according to credible theories in detail. Cosmology contains a variety of contents, so it is necessary to clarify the conventions and notations frequently used in the paper at first. The element of the space-time is given by

$$
d \mathbf{x}=\gamma_{\mu} d x^{\mu}=\gamma_{a} \delta X^{a},
$$


where $\gamma^{a}$ and $\gamma^{\mu}$ are tetrad expressed by Dirac matrices

$$
\gamma^{a}=\left(\begin{array}{cc}
0 & \tilde{\sigma}^{a} \\
\sigma^{a} & 0
\end{array}\right), \quad \gamma^{\mu}=h_{a}^{\mu} \gamma^{a}, \quad \gamma_{\mu}=l_{\mu}^{a} \gamma_{a},
$$

which satisfies the $C \ell_{1,3}$ Clifford algebra

$$
\gamma_{a} \gamma_{b}+\gamma_{b} \gamma_{a}=2 \eta_{a b}, \quad \eta_{a b}=\operatorname{diag}(1,-1,-1,-1), \quad \gamma_{\mu} \gamma_{\nu}+\gamma_{\nu} \gamma_{\mu}=2 g_{\mu \nu} .
$$

The Pauli matrices are expressed by

$$
\begin{aligned}
& \sigma^{a}=\left\{\left(\begin{array}{ll}
1 & 0 \\
0 & 1
\end{array}\right),\left(\begin{array}{ll}
0 & 1 \\
1 & 0
\end{array}\right),\left(\begin{array}{cc}
0 & -i \\
i & 0
\end{array}\right),\left(\begin{array}{cc}
1 & 0 \\
0 & -1
\end{array}\right)\right\}, \\
& \widetilde{\sigma}^{a}=\left(\sigma^{0},-\sigma^{1},-\sigma^{2},-\sigma^{3}\right), \quad \varrho^{\mu}=h^{\mu}{ }_{a} \sigma^{a}, \quad \widetilde{\varrho}_{\mu}=l_{\mu}^{a} \widetilde{\sigma}_{a},
\end{aligned}
$$

where $a, \mu \in\{0,1,2,3\}$. We take $c=1$ as unit of speed. For index, we use the Greek characters for curvilinear coordinates, Latin characters for local Minkowski coordinates and $\{i, j, k, l\}$ for spatial indices. The Einstein's summation is used.

Similarly to the case of metric $g_{\mu \nu}$, the definition of Ricci tensor can also differ by a negative sign. We take the definition as follows

$$
R_{\mu \nu} \equiv \partial_{\alpha} \Gamma_{\mu \nu}^{\alpha}-\partial_{\mu} \Gamma_{\nu \alpha}^{\alpha}-\Gamma_{\mu \beta}^{\alpha} \Gamma_{\nu \alpha}^{\beta}+\Gamma_{\mu \nu}^{\alpha} \Gamma_{\alpha \beta}^{\beta}, \quad R=g^{\mu \nu} R_{\mu \nu}
$$

In cosmology, the Lagrangian of main kinds of gravitational sources is generally given by

$$
\mathcal{L}=\frac{1}{2 \kappa}(R-2 \Lambda)+\mathcal{L}_{\mathrm{m}}, \quad \mathcal{L}_{\mathrm{m}}=\mathcal{L}_{\phi}+\mathcal{L}_{p}+\mathcal{L}_{\psi}+\mathcal{L}_{A}+\mathcal{L}_{\Phi}+\cdots,
$$

in which $\kappa=8 \pi G, \Lambda$ is the cosmological constant, $\mathcal{L}_{\mathrm{m}}$ the total Lagrangian of all matter.

$$
\begin{aligned}
\mathcal{L}_{\phi} & =\frac{1}{2} g^{\mu \nu} \partial_{\mu} \phi \partial_{\nu} \phi-V(\phi), \quad V \sim \frac{m^{2}}{2 k} \phi^{2 k} \\
\mathcal{L}_{p} & =-\sum_{n} m_{n} \sqrt{1-v_{n}^{2}} \delta^{3}\left(\vec{x}-\vec{X}_{n}\right), \quad v_{n}^{2}=g^{00} \bar{g}_{k l} \frac{d X_{n}^{k}}{d t} \frac{d X_{n}^{l}}{d t}, \\
\mathcal{L}_{\psi} & =\sum_{n}\left(\Re\left\langle\psi_{n}^{+} \alpha^{\mu} \hat{p}_{n \mid \mu} \psi_{n}\right\rangle-\psi_{n}^{+} \Omega_{\mu} \hat{S}^{\mu} \psi_{n}-m_{n} \psi_{n}^{+} \gamma^{0} \psi_{n}+N_{n}\right), \\
\mathcal{L}_{A} & =-\frac{1}{2} \nabla_{\mu} A_{\nu} \nabla^{\mu} A^{\nu}, \quad \mathcal{L}_{\Phi}=\frac{1}{2}\left(\nabla_{\mu} \Phi_{\nu} \nabla^{\mu} \Phi^{\nu}-b^{2} \Phi_{\mu} \Phi^{\mu}\right),
\end{aligned}
$$

where $\phi$ is the global slow-roll scalar field. $\mathcal{L}_{p}$ is the Lagrangian of ideal dust, whose statistical average is perfect fluid model. $v_{n}$ is the speed of $n$-th particle in usual sense. $\mathcal{L}_{\psi}$ is the Lagrangian for spinors with nonlinear potential $N_{n}$, electromagnetic potential $A^{\mu}$ and strong short distance interaction $\Phi^{\mu}$, in which the momentum operator

$$
\hat{p}_{k}^{\mu}=i\left(\hbar \partial^{\mu}+\Upsilon^{\mu}\right)-e_{k} A^{\mu}-s_{k} \Phi^{\mu}, \quad e_{k}=\{0, \pm e\}, \quad s_{k}=\{0, s\},
$$


where $\alpha^{\mu}$ is current operator and $\hat{S}^{\mu}$ spin operator. They are defined respectively by

$$
\alpha^{\mu}=\operatorname{diag}\left(\varrho^{\mu}, \widetilde{\varrho}^{\mu}\right), \quad \hat{S}^{\mu}=\frac{1}{2} \operatorname{diag}\left(\varrho^{\mu},-\widetilde{\varrho}^{\mu}\right) .
$$

$\Upsilon_{\mu} \in \Lambda^{1}$ is Keller connection, and $\Omega_{\mu} \in \Lambda^{3}$ is Gu-Nester potential. They are calculated by [27, 28, 29

$$
\Upsilon_{\mu}=\frac{1}{2} h_{a}^{\nu}\left(\partial_{\mu} l_{\nu}^{a}-\partial_{\nu} l_{\mu}^{a}\right), \quad \Omega^{\alpha}=\frac{1}{4} \epsilon^{d a b c} h_{d}^{\alpha} h_{a}^{\beta} S_{b c}^{\mu \nu} \partial_{\beta} g_{\mu \nu}, \quad S_{a b}^{\mu \nu} \equiv h_{[a}^{\{\mu} h_{b]}^{\nu\}} .
$$

In the Hamiltonian of a spinor we get a spin-gravity coupling energy $\hat{S}_{\mu} \Omega^{\mu}$. If the metric of the space-time can be orthogonalized, we have $\Omega_{\mu} \equiv 0$. In this paper we take the following simplest nonlinear potential as example to show its dynamical effects in cosmology

$$
N_{n}=\frac{1}{2} \widetilde{w} \check{\gamma}_{n}^{2}, \quad \check{\gamma}=\psi_{n}^{+} \gamma^{0} \psi_{n}
$$

The Hamiltonian formalism and classical mechanics can be clearly described only in the Gu's natural coordinate system 30

$$
d \mathbf{x}^{2}=g_{00} d t^{2}-\bar{g}_{k l} d x^{k} d x^{l}, \quad \sqrt{g}=\sqrt{g_{00}} \sqrt{\bar{g}}, \quad \bar{g}=\operatorname{det}\left(\bar{g}_{k l}\right),
$$

where $d \tau=\sqrt{g_{00}} d t$ defines the Newton's realistic cosmic time, which is different from the proper time of a particle $d s_{k}=\sqrt{1-v_{k}^{2}} d \tau$. The Dirac- $\delta$ is defined as

$$
\int_{\mathbb{R}^{3}} f(\vec{x}) \delta^{3}(\vec{x}-\vec{X}) \sqrt{\bar{g}} d^{3} x=f(\vec{X}), \quad \forall f \in C_{c}^{\infty}\left(\mathbb{R}^{3}\right),
$$

which means $\delta^{3}(\vec{x}-\vec{X}) \propto(\sqrt{\bar{g}})^{-1}$. Only scalar, spinor and vector fields can construct a proper Lagrangian[22], so (2.8)-(2.11) become the representation of all kinds of matter. In cosmology, to study them clearly is enough for theoretical analysis.

Variation of the Lagrangian (2.7) with respect to $g_{\mu \nu}$, we get Einstein's field equation

$$
G^{\mu \nu}+\Lambda g^{\mu \nu}+\kappa T^{\mu \nu}=0, \quad G^{\mu \nu} \equiv R^{\mu \nu}-\frac{1}{2} g^{\mu \nu} R=-\frac{\delta(R \sqrt{g})}{\sqrt{g} \delta g_{\mu \nu}} .
$$

where $\frac{\delta}{\delta g_{\mu \nu}}$ is the Euler derivatives, and $T^{\mu \nu}$ is EMT of all matter defined by

$$
T^{\mu \nu}=-2 \frac{\delta\left(\mathcal{L}_{\mathrm{m}} \sqrt{g}\right)}{\sqrt{g} \delta g_{\mu \nu}}=-2\left(\frac{\partial \mathcal{L}_{\mathrm{m}}}{\partial g_{\mu \nu}}-\left(\partial_{\alpha}+\Gamma_{\alpha \gamma}^{\gamma}\right) \frac{\partial \mathcal{L}_{\mathrm{m}}}{\partial\left(\partial_{\alpha} g_{\mu \nu}\right)}\right)-g^{\mu \nu} \mathcal{L}_{\mathrm{m}} .
$$

By calculation, we have 22, 28 .

$$
\begin{aligned}
T^{\mu \nu} & =T_{\phi}^{\mu \nu}+T_{p}^{\mu \nu}+T_{\psi}^{\mu \nu}+T_{A}^{\mu \nu}+T_{\Phi}^{\mu \nu}+\cdots, \\
T_{\phi}^{\mu \nu} & =\partial^{\mu} \phi \partial^{\nu} \phi-g^{\mu \nu}\left[\frac{1}{2} g^{\alpha \beta} \partial_{\alpha} \phi \partial_{\beta} \phi-V(\phi)\right], \\
T_{p}^{\mu \nu} & =\sum_{n} m_{n} u_{n}^{\mu} u_{n}^{\nu} \sqrt{1-v_{n}^{2}} \delta^{3}\left(\vec{x}-\vec{X}_{n}\right), \quad u_{n}^{\mu}=\frac{d}{d s} X_{n}^{\mu},
\end{aligned}
$$




$$
\begin{gathered}
T_{\psi}^{\mu \nu}=\sum_{n}\left(\frac{1}{2} \Re\left\langle\psi_{n}^{+}\left(\alpha^{\mu} \hat{p}_{n}^{\nu}+\alpha^{\nu} \hat{p}_{n}^{\mu}-2 S_{a b}^{\mu \nu} h_{c}^{\alpha} \eta^{b c} \alpha^{a} \hat{p}_{n \mid \alpha}\right) \psi_{n}\right\rangle\right. \\
\left.+\frac{1}{2} \epsilon^{a b c d} h^{\alpha}{ }_{a} h^{\beta}{ }_{b} S_{c d}^{\mu \nu} \check{S}_{n \mid \alpha ; \beta}+g^{\mu \nu} \Omega_{\alpha} \check{S}_{n}^{\alpha}+g^{\mu \nu} N_{n}\right), \\
T_{A}^{\mu \nu}=-\nabla^{\mu} A^{\alpha} \nabla^{\nu} A_{\alpha}+\frac{1}{2} g^{\mu \nu} \nabla_{\beta} A_{\alpha} \nabla^{\beta} A^{\alpha}, \\
T_{\Phi}^{\mu \nu}=\nabla^{\mu} \Phi^{\alpha} \nabla^{\nu} \Phi_{\alpha}-\frac{1}{2} g^{\mu \nu} \nabla_{\beta} \Phi_{\alpha} \nabla^{\beta} \Phi^{\alpha}+\frac{1}{2} b^{2}\left(\Phi^{\mu} \Phi^{\nu}+g^{\mu \nu} \Phi_{\alpha} \Phi^{\alpha}\right) .
\end{gathered}
$$

For classical approximation of (2.23), we have[31, 32]

$$
\left\{\begin{array}{l}
\psi^{+} \alpha^{\nu} \psi \rightarrow u^{\nu} \sqrt{1-v^{2}} \delta^{3}(\vec{x}-\vec{X}), \quad \hat{p}^{\mu} \psi \rightarrow m u^{\mu} \psi, \\
N \rightarrow w \sqrt{1-v^{2}} \delta^{3}(\vec{x}-\vec{X}), \quad \check{S}^{\alpha} \rightarrow S^{\alpha} \sqrt{1-v^{2}} \delta^{3}(\vec{x}-\vec{X}) .
\end{array}\right.
$$

Substituting (2.26) into (2.23) and noticing $S_{a b}^{\mu \nu}=-S_{b a}^{\mu \nu}$, we have

$$
\Re\left\langle\psi^{+} S_{a b}^{\mu \nu} h_{c}^{\alpha} \eta^{c b} \alpha^{a} \hat{p}_{\alpha} \psi\right\rangle \rightarrow m S_{a b}^{\mu \nu} u^{a} u^{b} \sqrt{1-v^{2}} \delta^{3}(\vec{x}-\vec{X})=0 .
$$

Omitting the tiny spin-gravity coupling energy, we get the classical approximation for EMT of dark spinor with self-interaction

$$
T_{\psi}^{\mu \nu} \rightarrow \sum_{n}\left(m_{n} u_{n}^{\mu} u_{n}^{\nu}+w_{n} g^{\mu \nu}\right) \sqrt{1-v_{n}^{2}} \delta^{3}\left(\vec{x}-\vec{X}_{n}\right),
$$

$w_{n} \propto(\sqrt{\bar{g}})^{-1}>0$ acts like negative pressure and takes the place of cosmological constant $\Lambda$ in equation (2.18). If $w_{n}=0$, the spinor moves along geodesic like a mass-point. Some researchers studied varying cosmological 'constant' $[33]$ - [40], but a directly varying $\Lambda(t)$ violates covariance of dynamics. The following calculation disclose that, the main physical origin of a covariant $\Lambda(a)$ may be just the nonlinear potential $\sum_{n} N_{n} \sim a^{-6}$.

For energy-momentum tensor, we have the following useful theorem, which means the energymomentum of any independent system is conserved respectively.

Theorem 1 Assume matter consists of two subsystems $I$ and II, namely $\mathcal{L}_{\mathrm{m}}=\mathcal{L}_{I}(\phi)+\mathcal{L}_{I I}(\psi)$, then we have

$$
T^{\mu \nu}=T_{I}^{\mu \nu}+T_{I I}^{\mu \nu}
$$

If the subsystems I and II have not interaction with each other, namely,

$$
\frac{\delta}{\delta \psi} \mathcal{L}_{I}(\phi)=\frac{\delta}{\delta \phi} \mathcal{L}_{I I}(\psi)=0
$$

then the two subsystems have independent energy-momentum conservation laws respectively,

$$
T_{I ; \nu}^{\mu \nu}=0, \quad T_{I I ; \nu}^{\mu \nu}=0 .
$$


Proof By the definition of EMT (2.19), the variation $\delta g: \mathcal{L}_{\mathrm{m}} \mapsto T^{\mu \nu}$ is a linear mapping, so (2.29) holds. By (2.30), the variables $\phi$ and $\psi$ have decoupling dynamic equations. Since the dynamics of variables is sufficient condition of energy-momentum conservation law, we can derive $T_{I ; \nu}^{\mu \nu}=0$ from dynamic equation of $\phi$, and $T_{I I ; \nu}^{\mu \nu}=0$ from dynamic equation of $\psi$ independently, so (2.31) holds. The proof is finished.

By Thm 1, we find in (2.8) the slow-roll scalar $\phi$ have not interaction with other components of matter, so we have $T_{\phi ; \nu}^{\mu \nu}=0$. In (2.9), each particle of ideal gas has not interaction with other components, so each particle satisfies energy-momentum conservation law. For $k$-th particle, by (2.22) we have

$$
\begin{aligned}
0 & =\int_{\mathbb{R}^{3}} T_{k ; \nu}^{\mu \nu} \sqrt{g} d^{3} x=\int_{\mathbb{R}^{3}}\left(\partial_{\nu}\left(T_{k}^{\mu \nu} \sqrt{g}\right)+\Gamma_{\alpha \beta}^{\mu} T_{k}^{\alpha \beta} \sqrt{g}\right) d^{3} x \\
& \rightarrow \frac{d}{d t}\left(\left(m_{k} u_{k}^{\mu} u_{k}^{0}\right) \sqrt{1-v_{k}^{2}} \sqrt{g_{00}}\right)+\Gamma_{\alpha \beta}^{\mu}\left(m_{k} u_{k}^{\alpha} u_{k}^{\beta}\right) \sqrt{1-v_{k}^{2}} \sqrt{g_{00}} .
\end{aligned}
$$

By $\frac{d s}{d t}=\sqrt{1-v_{k}^{2}} \sqrt{g_{00}}=\left(u_{k}^{0}\right)^{-1}$, we get geodesic equation for $k$-th particle

$$
\frac{d}{d s} u_{k}^{\mu}+\Gamma_{\alpha \beta}^{\mu} u_{k}^{\alpha} u_{k}^{\beta}=0 .
$$

For ideal mass point, energy momentum conservation law is equivalent to dynamics. For perfect fluid model, the conclusion is also right. However, the Lagrangian should also be (2.9).

If $e_{k} \neq 0$ or $s_{k} \neq 0$, spinor $\psi_{k}$ interacts with $A^{\mu}$ or $\Phi^{\mu}$, so we should take it as one system. For an isolated particle, the classical approximation of static $A^{\mu}$ or $\Phi^{\mu}$ of the particle can be treated as an additional mass $\delta m_{k}$ due to linearity of $A^{\mu}$ and $\Phi^{\mu}$. We calculate $\delta m_{k}$ in the next section. The propagating $A^{\mu}$ is photon, which can be treated as massless particles. Thus except the global scalar $\phi$, the classical approximation of EMT for other fields can be generally depicted by

$$
T^{\mu \nu} \rightarrow(\rho+P) U^{\mu} U^{\nu}+(W-P) g^{\mu \nu}
$$

The statistical expectation of nonlinear potentials is function of state $W(\rho)$ which acts like negative pressure. By (2.15) we find $W_{\psi} \sim\left(\delta^{3}(\vec{x})\right)^{2}$ is scale dependent. We have $W_{\psi} \propto \rho(\sqrt{\bar{g}})^{-1} \propto(\sqrt{\bar{g}})^{-2}$.

\section{EQUATION OF STATE IN COSMOLOGY}

\section{A. Basic Equations in Cosmology}

In cosmology, we have Friedmann-Lemaitre-Robertson-Walker(FLRW) metric

$$
d s^{2}=d \tau^{2}-a(\tau)^{2}\left(d r^{2}+\mathcal{S}(r)^{2}\left(d \theta^{2}+\sin ^{2} \theta d \phi^{2}\right)\right),
$$


in which $\mathcal{S}(r)=\sin r, r, \sinh r$ correspond to $K=1,0,-1$ respectively. By symmetry we get

$$
\begin{aligned}
& G_{\tau}^{\tau}=-\frac{3}{a^{2}}\left(a^{\prime 2}+K\right), \quad R=\frac{6}{a^{2}}\left(a a^{\prime \prime}+a^{\prime 2}+K\right), \\
& G_{r}^{r}=G_{\theta}^{\theta}=G_{\varphi}^{\varphi}=-\frac{1}{a^{2}}\left(2 a a^{\prime \prime}+a^{\prime 2}+K\right), \quad a^{\prime}=\frac{d}{d \tau} a(\tau) .
\end{aligned}
$$

For all gravitational sources $\mathcal{L}_{\mathrm{m}}$ we have EMT in traditional form

$$
T_{\nu}^{\mu}=\operatorname{diag}\left(\rho_{\mathrm{m}},-P_{\mathrm{m}},-P_{\mathrm{m}},-P_{\mathrm{m}}\right) .
$$

The dynamics of cosmology $\left\{(2.18) \& T_{; \nu}^{\mu \nu}=0\right\}$ are overdetermined due to symmetry of FLRW metric. It is easy to check, among all dynamical equations, only the following equations are independent equations, but the spatial components of the Einstein's field equations can be derived from these equations. So we have dynamics for FLRW universe as

$$
\begin{aligned}
G_{\tau}^{\tau}+\Lambda+\kappa T_{\tau}^{\tau} & =0 \Leftrightarrow a^{\prime 2}=\frac{1}{3} \Lambda a^{2}-K+\frac{\kappa}{3} \rho_{\mathrm{m}} a^{2} . \\
T_{i ; \nu}^{\mu \nu} & =0 \Leftrightarrow d\left(\rho_{i} a^{3}\right)=-P_{i} d a^{3}, \quad(\forall i),
\end{aligned}
$$

where index $i$ means independent subsystem. All other equations can be derived from the above equations. Together with initial values and equations of state $P_{i}=P_{i}\left(\rho_{i}\right)$, the above equations are closed and the solution is uniquely determined.

The analysis in the next section shows that the asymptotic property of $P$ as $a \rightarrow+0$ is crucial for the fate of the universe, so we give detailed discussion on this problem. At first, we examine the ideal gases. To solve the geodesic of the particles, we prove a useful theorem.

Theorem 2 Assume the line element of a manifold has the following form

$$
d s^{2}=a(t)^{2} d t^{2}+g_{\mu \nu}(t) d x^{\mu} d x^{\nu}, \quad \mu, \nu \in\{1,2, \cdots, n\},
$$

then the geodesic in the manifold is integrable,

$$
\frac{d}{d s} t=\frac{1}{a} \sqrt{1-g^{\mu \nu} b_{\mu} b_{\nu}}, \quad \frac{d}{d s} x^{\mu}=g^{\mu \nu} b_{\nu}
$$

where $b_{\mu}$ are integral constants.

Proof The nonzero christoffel symbols are $\Gamma_{t t}^{t}, \Gamma_{\mu \nu}^{t}$ and

$$
\Gamma_{t \nu}^{\mu}=\Gamma_{\nu t}^{\mu}=\frac{1}{2} g^{\mu \alpha} g_{\alpha \nu}^{\prime}, \quad(\mu, \nu \neq 0),
$$

where $g_{\alpha \nu}^{\prime}=\frac{d}{d t} g_{\alpha \nu}, g^{\mu \alpha} g_{\alpha \nu}=\delta_{\nu}^{\mu}$. Since $\frac{d}{d s} t$ can be directly derived from (3.7), it is unnecessary to calculate $\Gamma_{t t}^{t}$ and $\Gamma_{\mu \nu}^{t}$. Denoting $\dot{x}^{\mu}=\frac{d}{d s} x^{\mu}$, the geodesic equation of $x^{\mu}$ is given by

$$
\ddot{x}^{\mu}+2 \Gamma_{0 \nu}^{\mu} \dot{t} \dot{x}^{\nu}=\ddot{x}^{\mu}+g^{\mu \alpha} g_{\alpha \nu}^{\prime} \dot{t}^{\nu}=0 .
$$


Multiplying $g_{\mu \beta} \dot{t}^{-1}$ on both sides we get

$$
g_{\mu \beta} \frac{d}{d t} \dot{x}^{\mu}+\frac{d}{d t}\left(g_{\beta \nu}\right) \dot{x}^{\nu}=\frac{d}{d t}\left(g_{\mu \beta} \dot{x}^{\mu}\right)=0 .
$$

The solution is given by

$$
g_{\mu \nu} \dot{x}^{\nu}=b_{\mu}, \quad \dot{x}^{\mu}=g^{\mu \nu} b_{\nu},
$$

where $b_{\nu}$ is integral constant. By (3.7) and $(\overline{3.12})$ we get

$$
\dot{t}^{2}=a^{-2}\left(1-g_{\mu \nu} \dot{x}^{\mu} \dot{x}^{\nu}\right)=a^{-2}\left(1-g^{\mu \nu} b_{\mu} b_{\nu}\right),
$$

so (3.8) holds. The proof is finished.

For the FLRW metric (3.1), the line element of orthogonal subspace-time $(\tau, r)$ has the form of (3.7), so the geodesic in this subspace-time can be solved as

$$
\frac{d r}{d s}=\frac{C}{a^{2}}, \quad \frac{d \tau}{d s}=\frac{1}{a} \sqrt{a^{2}+C^{2}},
$$

where $C$ is a constant determined by initial data.

\section{B. Equation of State of Ideal gases}

By (3.14) we get the drifting speed of $n$-th particle in usual sense

$$
v_{n} \equiv \frac{a d r}{d \tau}=\frac{b_{n}}{\sqrt{a^{2}+b_{n}^{2}}}, \quad \sqrt{1-v_{n}^{2}}=\frac{a}{\sqrt{a^{2}+b_{n}^{2}}} .
$$

So the momentum of the particle satisfies

$$
p(\tau) a(\tau)=p\left(\tau_{0}\right) a\left(\tau_{0}\right)=C, \quad p=\frac{m_{n} v_{n}}{\sqrt{1-v_{n}^{2}}},
$$

where $m_{n}$ is the proper mass of the particle. For the massless photons, it is easy to check that the wavelength $\lambda(\tau) \propto a(\tau)$, so their momentum $p$ also satisfy (3.16). Although (3.16) is derived in subspace-time $(\tau, r)$, but it is suitable for all particles due to the symmetry of the FLRW metric.

Now we establish the relation between the state functions $\left(\rho_{p}, P_{p}\right)$ of the ideal gases and scale factor $a$. By (3.16) we have $p_{n}^{2}=C_{n} a^{-2}$, where $C_{n}$ are constants determined by initial data at $\tau=\tau_{0}$. Then on one hand, for all particles we have the mean square momentum as

$$
\bar{p}^{2}=\frac{C_{0}}{a^{2}},
$$


where $C_{0}$ is a constant only determined by initial data at $\tau_{0}$. On the other hand, the relativistic relation between momentum $p$ and the kinetic energy $\mathcal{K}$ is given by

$$
p^{2}=\mathcal{K}(\mathcal{K}+2 m), \quad \mathcal{K}=\frac{m}{\sqrt{1-v^{2}}}-m,
$$

so $\bar{p}^{2}$ can be also calculated by statistics. Assuming the distribution of kinetic energy $\mathcal{K}$ of the ideal gases is given by $d \mathcal{P}=\mathcal{F}(\mathcal{K}) d \mathcal{K}$. Then it satisfies the equation of moments

$$
\int_{0}^{\infty} \mathcal{F}(\mathcal{K}) d \mathcal{K}=1, \quad \int_{0}^{\infty} \mathcal{K} d \mathcal{P}=\frac{3}{2} k T, \quad \int_{0}^{\infty} \mathcal{K}^{2} d \mathcal{P}=\frac{3}{2 \sigma}(k T)^{2}
$$

where the second formula is the definition of temperature $T$ of the gases, $k=1.38 \times 10^{-23} \mathrm{~J} / \mathrm{K}$ is the Boltzmann constant, $\sigma \sim \frac{2}{5}$ is a constant reflecting the distribution function of particles. In the case of Maxwell distribution [41], we have $\sigma=\frac{2}{5}$.

By (3.18) and the moments (3.19) we have

$$
\bar{p}^{2}=\frac{1}{N} \sum_{n=1}^{N} \int_{0}^{\infty} p_{n}^{2} \mathcal{F}\left(\mathcal{K}_{n}\right) d \mathcal{K}_{n}=\frac{3}{2 \sigma} k T(k T+2 \sigma \bar{m}),
$$

where $\bar{m}=\frac{1}{N} \sum_{n} m_{n}$ is average proper mass of all particles. Comparing (3.20) with (3.17), we get the relation $T(a)$,

$$
k T=\frac{\sigma \bar{m}}{a}\left(\sqrt{a^{2}+b^{2}}-a\right), \quad a=\frac{\sigma b \bar{m}}{\sqrt{k T(k T+2 \sigma \bar{m})}},
$$

where $b>0$ is a constant determined by initial data. We take $a$ as independent variable in statistical calculation. Likewise, by (2.22) and (3.21) we have

$$
\begin{aligned}
\rho_{p} & \equiv \sum_{n} \frac{m_{n}}{\sqrt{1-v_{n}^{2}}} \delta^{3}\left(\vec{x}-\vec{X}_{n}\right)=\frac{1}{V} \int_{V} \sum_{X_{n} \in V} \frac{m_{n}}{\sqrt{1-v_{n}^{2}}} \delta^{3}\left(\vec{x}-\vec{X}_{n}\right) \sqrt{\bar{g}} d^{3} x \\
& =\frac{1}{a^{3} \Omega} \sum_{X_{n} \in \Omega} \int \frac{m_{n}}{\sqrt{1-v_{n}^{2}}} d \mathcal{P}=\frac{1}{a^{3} \Omega} \sum_{X_{n} \in \Omega} \int\left(m_{n}+\mathcal{K}_{n}\right) d \mathcal{P} \\
& =\frac{1}{a^{3} \Omega} \sum_{X_{n} \in \Omega}\left(\bar{m}+\frac{3}{2} k T\right)=\frac{\varrho}{a^{3}}\left(1+\frac{3 \sigma}{2 a}\left(\sqrt{a^{2}+b^{2}}-a\right)\right),
\end{aligned}
$$

where $V=a^{3} \Omega, \varrho=\frac{1}{\Omega} \sum_{X_{n} \in \Omega} m_{n}$ is the angular density of proper mass, which is a constant. Substituting (3.22) into energy conservation law (3.6) we get

$$
P_{p} \equiv \frac{1}{3} \sum_{n} \frac{m_{n} v_{n}^{2}}{\sqrt{1-v_{n}^{2}}} \delta^{3}\left(\vec{x}-\vec{X}_{n}\right)=\frac{\sigma \varrho b^{2}}{2 a^{4} \sqrt{a^{2}+b^{2}}}=\frac{N k T}{V}\left(1-\frac{k T}{2(\sigma \bar{m}+k T)}\right) .
$$

While $a \rightarrow+0$ we find $\rho_{p} \sim P_{p} \rightarrow C a^{-4}$. Ideal gases cannot provide negative pressure. 
In the above derivation, the FLRW metric (3.1) is used as piston-cylinder system to drive the ideal gas, and we can prove that the elastic collisions of particles have no influence on these results[41. So the results are actually valid in general cases. By (3.21) we have relation

$$
a=\frac{\sigma b}{\sqrt{J(J+2 \sigma)}}, \quad J \equiv \frac{k T}{\bar{m} c^{2}}=\frac{\sigma}{a}\left(\sqrt{a^{2}+b^{2}}-a\right),
$$

where $J$ is dimensionless temperature. The above results conclude the following theorem.

Theorem 3 For relativistic ideal gas, we have the EOS as

$$
P=\frac{\bar{\rho} k T}{\bar{m} c^{2}}\left(1-\frac{k T}{2\left(\sigma \bar{m} c^{2}+k T\right)}\right), \quad \bar{m} \equiv \frac{1}{N} \sum_{n=1}^{N} m_{n}, \quad \bar{\rho} \equiv \frac{N \bar{m}}{V} \propto \frac{1}{a^{3}} .
$$

For adiabatic process the functions of state satisfy parameter equation

$$
\bar{\rho}=\rho_{0}[J(J+2 \sigma)]^{\frac{3}{2}}, \quad \rho=\bar{\rho}\left(1+\frac{3}{2} J\right), \quad P=\bar{\rho} \frac{J(J+2 \sigma)}{2(J+\sigma)},
$$

where $J$ acts as independent variable, $\rho_{0}=\varrho(\sigma b)^{-3}$ is a constant density.

The above derivation is compatible with relativity and includes the driving effect of gravity. In the case of low temperature, Thm $\sqrt[3]{3}$ gives the EOS for the adiabatic monatomic gas

$$
P \doteq \rho J \doteq P_{0} \rho^{\frac{5}{3}}, \quad(J \ll 1, \text { or } k T \ll \bar{m}),
$$

which is identical to the empirical law of thermodynamics. Letting $J \rightarrow \infty$ or $\bar{m} \rightarrow 0$, we get the Stefan-Boltzmann's law $\rho \propto T^{4}$. Thus the above results are automatically suitable for photons, and the Stefan-Boltzmann's law is also valid for the ultra-relativistic particles. In general relativity, all processes occur automatically, and $\rho_{0}$ is independent of any practical process.

\section{Asymptotic Behavior of Scalar field in the Early Universe}

For scalar field $\phi$, by (2.21) we have

$$
\mathcal{L}_{\phi}=\frac{1}{2} \phi^{\prime 2}-V, \quad \rho_{\phi}=\frac{1}{2} \phi^{\prime 2}+V, \quad P_{\phi}=\frac{1}{2} \phi^{\prime 2}-V .
$$

For potential $V=\frac{1}{2 k} m^{2} \phi^{2 k},(k \geq 1)$, by variation with respect to $\phi$ we get dynamical equation

$$
\phi^{\prime \prime}+\frac{3 a^{\prime}}{a} \phi^{\prime}+m^{2} \phi^{2 k-1}=0 .
$$

(3.29) can be also derived by substituting (3.28) into energy conservation law (3.6). If the universe has an initial singularity, we set $a(0)=0$. For low temperature particles, we have $\rho_{p} a^{3} \sim$ const. By Friedmann equation (3.5), we find $a \geq a_{0} \sqrt[3]{\tau^{2}}$ while $\tau \rightarrow+0$. So we assume

$$
a \rightarrow a_{0} \tau^{j}, \quad \phi \rightarrow \frac{C_{0}}{\tau^{i}}, \quad\left(j \sim \frac{2}{3}, j<1, i>0\right) .
$$


About the range of $j$, Barrow made some analysis in [42]. Substituting (3.30) into (3.29) we get solution in the case of $k>1$,

$$
\begin{aligned}
i & =\frac{1}{k-1}, \quad m^{2}=\frac{3 j(k-1)-k}{C_{0}^{2(k-1)}(k-1)^{2}}, \quad j>\frac{k}{3(k-1)} \\
\rho_{\phi} & \rightarrow \frac{3 j C_{0}^{2}}{2 k(k-1)} \tau^{-\frac{2 k}{k-1}}, \quad P_{\phi} \rightarrow-\frac{3 j(k-1)-2 k}{2 k(k-1)^{2}} C_{0}^{2} \tau^{-\frac{2 k}{k-1}} .
\end{aligned}
$$

Substituting $a \rightarrow a_{0} \tau^{j}$ into (3.32), we get

$$
P_{\phi} \rightarrow\left(\frac{2 k}{3(k-1)}-j\right) \frac{C^{2}}{a^{n}}, \quad(2<n<6, a \rightarrow+0) .
$$

In the case of $k=1,(\overline{3.29})$ is a linear equation of $\phi$. Substituting $a \rightarrow a_{0} \tau^{j}$ into (3.29), we get

$$
\phi \rightarrow \tau^{-\alpha}\left[C_{1} J_{\alpha}(m \tau)+C_{2} Y_{\alpha}(m \tau)\right] \rightarrow C_{3} \tau^{1-3 j}, \quad \alpha=\frac{1}{2}(3 j-1),
$$

where $(J, Y)$ are Bessel functions. $\phi \rightarrow C_{4} \ln \tau$ while $\alpha=0$. Substituting the above results into (3.28) we get

$$
\rho_{\phi} \sim P_{\phi} \rightarrow \frac{C^{2}}{\tau^{6 j}}=\frac{C^{2}}{a^{6}}>0, \quad(a \rightarrow+0) .
$$

If $V(\phi)$ is the polynomial of $\phi$, the asymptotic property depends only on the highest power, so (3.33) and (3.35) are valid for all cases. Substituting $\rho_{\phi} \rightarrow C^{2} a^{-n}$ into Friedmann equation (3.5) we get

$$
a^{\prime 2} \geq C_{5}^{2} a^{2-n} \Leftrightarrow a \geq a_{0} \tau^{\frac{2}{n}}, \quad(a \rightarrow+0) .
$$

By $2<n \leq 6$ we find $\frac{1}{3} \leq j<1$. For the linear scalar $\phi$, by $(3.35)$ we find it cannot provide negative pressure for the early universe. For the nonlinear $\phi$, by $(\overline{3.33})$ we find $P_{\phi}<0$ only if $j>\frac{2 k}{3(k-1)}$. By the constraint $\frac{1}{3} \leq j<1, \phi$ can provide negative pressure only for $k>3$. So taking scalar field $\phi$ as main reason to explain the accelerating expansion of the early universe is grudging and unnatural.

\section{Equation of State of Spinor Gas}

The above calculation shows that perfect fluid and scalar model cannot provide negative pressure. Now we examine the dark spinor with nonlinear potential. In this case, the classical EMT is defined by (2.28). The spinor moves approximately along geodesic, i.e., for nonlinear spinors the equivalence principle holds approximately, so the functions of state $\left(\rho_{\psi}, P_{\psi}\right)$ should approximately 
equal $\left(\rho_{p}, P_{p}\right)$ given by (3.22) and (3.23). We only need to derive the function $W_{\psi}$. By (2.28), the definition of $W_{\psi}$ in microscopic view reads

$$
W_{\psi} \equiv \frac{1}{V} \int_{V} \sum_{n} w_{n} \delta^{3}\left(\vec{x}-\vec{X}_{n}\right) \sqrt{1-v_{n}^{2}} d V=\frac{1}{V} \sum_{X_{n} \in V} w_{n} \sqrt{1-v_{n}^{2}}
$$

The statistical expectation of $\sqrt{1-v_{n}^{2}}$ cannot be directly calculated according to moments (3.19). By (3.15), we get

$$
\sqrt{1-v_{n}^{2}}=\frac{b_{n}}{\sqrt{a^{2}+b_{n}^{2}}}=\frac{d}{d a} \frac{a}{\sqrt{1-v_{n}^{2}}}=\frac{1}{m_{n}} \frac{d}{d a}\left[a\left(m_{n}+\mathcal{K}_{n}\right)\right] .
$$

Substituting it into (3.37) and using (3.21), we get expectation value

$$
\begin{aligned}
W_{\psi} & =\frac{1}{V} \sum_{X_{n} \in V} \int w_{n} \sqrt{1-v_{n}^{2}} d \mathcal{P}=\frac{1}{V} \sum_{X_{n} \in V} \frac{w_{n}}{m_{n}} \frac{d}{d a} \int a\left(m_{n}+\mathcal{K}_{n}\right) d \mathcal{P} \\
& =\frac{1}{V} \sum_{X_{n} \in V} \frac{w_{n}}{m_{n}} \frac{d}{d a}\left(a\left(m_{n}+\frac{3}{2} k T\right)\right) \\
& =\frac{1}{V} \sum_{X_{n} \in V}\left(w_{n}-\frac{3 w_{n}}{2 m_{n}} \sigma \bar{m}\left(1-\frac{a}{\sqrt{a^{2}+b^{2}}}\right)\right) \\
& =\bar{\rho}\left(\frac{\bar{w}}{\bar{m}}-\frac{3 \mu \sigma k T}{2(\sigma \bar{m}+k T)}\right),
\end{aligned}
$$

where the mean parameters are defined by

$$
\bar{w}=\frac{1}{N} \sum_{n=1}^{N} w_{n} \rightarrow \frac{C_{1}}{a^{3}}, \quad \mu=\frac{1}{N} \sum_{n=1}^{N} \frac{w_{n}}{m_{n}} \rightarrow \frac{C_{2}}{a^{3}}, \quad(a \rightarrow+0) .
$$

By (3.24) and (3.26) we get the dimensionless form,

$$
W_{\psi}=\bar{\rho}\left(\frac{\bar{w}}{\bar{m}}-\frac{3 \mu \sigma J}{2(J+\sigma)}\right)=\frac{\varrho}{a^{3}}\left(\frac{\bar{w}}{\bar{m}}-\frac{3 \mu \sigma\left(\sqrt{a^{2}+b^{2}}-a\right)}{2 \sqrt{a^{2}+b^{2}}}\right) \rightarrow \frac{C}{a^{6}} .
$$

For nonlinear spinors, by (2.34) we have

$$
T_{\tau \mid \psi}^{\tau}=\rho_{\psi}+W_{\psi}, \quad T_{r \mid \psi}^{r}=T_{\theta \mid \psi}^{\theta}=T_{\varphi \mid \psi}^{\varphi}=W_{\psi}-P_{\psi}
$$

So its EOS in cosmology is given by 43 .

$$
w_{\psi}(a) \equiv-\frac{T_{r \mid \psi}^{r}}{T_{\tau \mid \psi}^{\tau}}=\frac{P_{\psi}-W_{\psi}}{\rho_{\psi}+W_{\psi}} \rightarrow-1, \quad(a \rightarrow+0) .
$$

The above results suggest that dark nonlinear spinors may be the real dark energy and dark matter, which determines the large scale structure of the universe. We confirm this conclusion in the next section. 
Now we consider the classical approximation of EMT (2.24) of electromagnetic field. We have two types of energy-momentum for $A^{\mu}$. The propagating photon can be directly treated as a particle with $P=\frac{1}{3} \rho$. The stationary electromagnetic field of $\psi_{k}$ satisfies

$$
\partial_{\mu} \partial^{\mu} A_{k}^{\nu}=e_{k} \check{\alpha}_{k}^{\nu}, \quad \check{\alpha}_{k}^{\nu} \equiv \psi_{k}^{+} \alpha^{\nu} \psi_{k} .
$$

By the principle of superposition, we have $A^{\mu}=\sum_{k} A_{k}^{\mu}$. (3.45) in the comoving coordinate system with natural boundary condition $\left(A^{\mu} \rightarrow 0, r \rightarrow \infty\right)$ is static electromagnetic field, which can be solved by means of Green function. Then the general solution of (3.45) can be derived from static solution by local Lorentz transformation. We calculate the classical approximation of EMT (2.24) concretely.

The stationary field $A^{\mu}$ has only a very tiny geometrical structure like a Dirac- $\delta$, so we can omit the effect of curved space-time in comoving frame and calculate it in tangent Minkowski spacetime. For simplicity, we take one particle as example to calculate EMT, and omit the particle index temporarily. By local Lorentz transformation we have[32]

$$
T^{a b}(\mathbf{x})=\sqrt{1-v^{2}} L_{c}^{a} L_{d}^{b} \bar{T}^{c d}(\mathbf{X}), \quad d x^{a}=L_{b}^{a} d X^{b} .
$$

where $\mathbf{X}=\gamma_{a} X^{a}$ is the comoving coordinate of the particle, $L_{b}^{a}$ is Lorentz transformation, and $\bar{T}^{c d}(\mathbf{X})$ is the static EMT of the spinor $\psi$.

In the comoving coordinate system, we have classical approximation

$$
\bar{T}^{a b}(\mathbf{X}) \rightarrow \delta^{3}(\vec{X}) \int_{\mathbb{R}^{3}}\left(-\partial^{a} A_{c} \partial^{b} A^{c}+\frac{1}{2} \eta^{a b} \partial_{c} A_{d} \partial^{c} A^{d}\right) d^{3} X .
$$

For static charge, we have $\partial_{0} A^{a} \equiv 0$, and then $\bar{T}^{0 k} \equiv 0$,

$$
\begin{aligned}
\bar{T}^{00}(\mathbf{X}) & \rightarrow-\frac{1}{2} \delta^{3}(\vec{X}) \int_{\mathbb{R}^{3}}\left(\nabla A_{d}\right) \cdot\left(\nabla A^{d}\right) d^{3} X \\
& =\frac{1}{2} \delta^{3}(\vec{X}) \int_{\mathbb{R}^{3}} A_{a} \Delta A^{a} d^{3} X=-\frac{1}{2} \delta^{3}(\vec{X}) \int_{\mathbb{R}^{3}} e A_{a} \check{\alpha}^{a} d^{3} X \\
& =-\frac{e^{2}}{8 \pi} \delta^{3}(\vec{X}) \int_{\mathbb{R}^{6}} \frac{\check{\alpha}_{a}(\vec{\xi}) \check{\alpha}^{a}(\vec{X})}{|\vec{\xi}-\vec{X}|} d^{3} \xi d^{3} X=-m_{e} \delta^{3}(\vec{X}),
\end{aligned}
$$

in which $m_{e}$ is the proper electromagnetic mass of the particle defined by

$$
m_{e}=\frac{e^{2}}{8 \pi} \int_{\mathbb{R}^{6}} \frac{\check{\alpha}_{a}(\vec{\xi}) \check{\alpha}^{a}(\vec{X})}{|\vec{\xi}-\vec{X}|} d^{3} \xi d^{3} X .
$$

Different from the nonlinear potential, $m_{e}$ is a constant independent of scale.

By symmetry of $\check{\alpha}^{a}(\vec{X})$, we have $\bar{T}^{k l}=0,(k \neq l)$, so $\bar{T}^{a b}$ is diagonal.

$$
\bar{T}_{a}^{a}(\mathbf{X}) \rightarrow \delta^{3}(\vec{X}) \int_{\mathbb{R}^{3}}\left(-\partial_{a} A_{b} \partial^{a} A^{b}+2 \partial_{c} A_{d} \partial^{c} A^{d}\right) d^{3} X=-2 \bar{T}_{0}^{0}=2 m_{e} \delta^{3}(\vec{X}) .
$$


Thus, in comoving coordinate system we get average classical approximation of EMT as

$$
\bar{T}_{b}^{a}(\mathbf{X}) \rightarrow m_{e} \delta^{3}(\vec{X}) \operatorname{diag}(-1,1,1,1),
$$

which provided negative energy and negative pressure. Converting (3.51) into general form we get

$$
T_{A}^{\mu \nu}(\mathbf{x}) \rightarrow \sum_{k} m_{e k}\left(-2 u_{k}^{\mu} u_{k}^{\nu}+g^{\mu \nu}\right) \sqrt{1-v_{k}^{2}} \delta^{3}\left(\vec{x}-\vec{X}_{k}\right)
$$

From $\hat{p}_{\psi}^{\mu}$ defined in (2.12), we find $\psi_{k}$ and $A_{k}^{\mu}$ are not independent systems, and some EMT components of electromagnetic field are included in $T_{\psi}^{\mu \nu}$ given by (2.23). Therefore, the EMT (3.52) of stationary $A^{\mu}$ can be merged into $T_{\psi}^{\mu \nu}$, and we don't need to calculate it separately. The situation of field $\Phi$ is the same, we don't discuss it any more.

From the discussion of this section, we get some important knowledge about the total mass density $\rho_{\mathrm{m}}=T_{0}^{0}$ and the total pressure $P_{\mathrm{m}}=-\frac{1}{3} T_{k}^{k}$. Except for the global scalar field $\phi$, the classical approximation of EMT of all other matter has a standard formalism (2.34), and the EOS is algebra equation. By local structure in the universe such as galaxies, the uniform scalar $\phi$ is obviously much weaker than other kind components of matter. While $a \rightarrow+0, P_{\mathrm{m}}$ is controlled by function $W \sim-\mathrm{O}\left(a^{-6}\right)[28,43]$. In cosmology, although we call $P_{\mathrm{m}}$ pressure, but it is actually a variable including the potentials of all fields [11, 21, 22]. In general, we have conclusions:

$1^{\circ}$ The total mass-energy density is always positive, namely

$$
\rho_{\mathrm{m}}>0, \quad(\forall a>0)
$$

$2^{\circ}$ The total pressure $P_{\mathrm{m}}<0$ when the universe is small enough, namely

$$
P_{\mathrm{m}}<0, \quad(a \rightarrow+0) .
$$

The introduction of scalar $\phi$ is purely an artificial design, which has little use for clarifying the mysteries of the universe. According to the analysis of literatures [22, 32], the physical and logical reason for the existence of scalars is limited. The following discussion also reveals, $\phi$ has little influence on the behavior of the universe.

\section{DYNAMICAL CONSTRAINTS ON $K$ AND $\Lambda$}

Since the Friedmann equation is a dynamical equation, it is hard to determine its constants by static analysis. In [12, 18, 23, 24], under respective assumptions the authors have provided some dynamical analysis for the behavior of the universe. Here we also qualitatively analyze the 
dynamical properties of the Friedmann equation according to the above preliminaries. Under the assumptions of positive mass density (3.53) and negative initial pressure (3.54), we find that the universe cannot reach the initial singularity, as well as the parameters $K=1$ and $\Lambda \simeq 0$. That is to say, the spatial structure of the universe is closed 3 dimensional sphere $S^{3}$, and the cosmological constant is likely to be zero. Besides, the universe should be cyclic in time. Obviously, these conclusions will help to correct some popular misconceptions and bring great convenience to further study the properties of other problems in cosmology such as dark matter and dark energy.

From the previous analysis, we find that the scale factor $a(\tau) \sim \mathrm{O}\left(\tau^{j}\right)$ is nonanalytic at origin, which increases difficulty for analysis, so we adopt the conformal FLRW metric,

$$
d s^{2}=a(t)^{2}\left(d t^{2}-d r^{2}-\mathcal{S}(r)^{2}\left(d \theta^{2}+\sin ^{2} \theta d \phi^{2}\right)\right) .
$$

Then the Friedmann equation (3.5) becomes

$$
a^{\prime 2}=-K a^{2}+\frac{1}{3} \Lambda a^{4}+\frac{1}{3} \kappa \rho_{\mathrm{m}} a^{4} .
$$

(4.2) can be rewritten as

$$
a^{\prime 2}=F(a), \quad F(a) \equiv 2 \bar{R} a-K a^{2}+\frac{1}{3} \Lambda a^{4}+X(a),
$$

where $\bar{R}$ corresponds to the total conformal density of proper mass,

$$
\bar{R} \equiv \lim _{a \rightarrow \infty} \frac{1}{6} \kappa \rho_{\mathrm{m}} a^{3} \approx \frac{1}{6} \kappa \varrho
$$

which is a constant. The physical meaning of (4.4) means the total conformal energy of the universe is bounded. By (4.3) we find $\bar{R}$ has length dimension, which is the mean scale of the universe[21].

Comparing (4.3) with (4.2), we get relation

$$
\begin{aligned}
\rho_{\mathrm{m}} & =\frac{3}{\kappa a^{4}}\left(F(a)+K a^{2}-\frac{1}{3} \Lambda a^{4}\right) \\
& =\frac{3}{\kappa a^{4}}[2 \bar{R} a+X(a)] .
\end{aligned}
$$

$X(a)$ corresponds to the rest and unknown parts of $\rho_{\mathrm{m}}$, which satisfies $X(a) \sim-\mathrm{O}\left(a^{-3}\right)$ as $a \rightarrow+0$ and $X(a) / a \rightarrow 0$ as $a \rightarrow \infty$. In physics, $X(a)$ is mainly determined by nonlinear potential $\sum_{k} N_{k}$. The specific form of $X(a)$ is not important for qualitative analysis, only its asymptotic properties as $a \rightarrow+0$ have influence on the following discussion. The property of the solution of (4.3) can be clearly discussed by means of phase trajectories.

Substituting (4.6) into energy conservation law (3.6), we get the total pressure as

$$
P_{\mathrm{m}}=-\frac{1}{\kappa a^{4}}\left[X^{\prime}(a) a-X(a)\right] .
$$


We find $P_{\mathrm{m}}$ is irrelative to $\bar{R}$. Since the derivatives of pressure and potential correspond to ordinary forces which should be finite, so $P_{\mathrm{m}}$ should be at least continuous. Then by (4.7) we have at least $X(a) \in C^{1}$. Consequently, by the definition of $F(a)$ in (4.3), we also have $F(a) \in C^{1}$.

The following discussion is based on Friedamnn equation (4.3) as well as two assumptions (3.53) and (3.54), namely, the positive total energy density $\rho_{\mathrm{m}}>0$ and negative initial pressure $P_{\mathrm{m}}(a)<0,(a \rightarrow+0)$. Clearly the two assumptions are compatible with observational facts[24].

Theorem 4 For function $F(a) \in C^{1}$ defined in (4.3), if $\lim _{a \rightarrow 0} X(a) \neq 0$ and condition (3.54) holds, we have

$$
F(a)<0, \quad(a \rightarrow+0) .
$$

Proof In the case of $0<|X(0)|<\infty$, by (4.7) and condition (3.54), we have

$$
P_{\mathrm{m}} \rightarrow \frac{X(0)}{\kappa a^{4}}<0, \quad(a \rightarrow+0) .
$$

Consequently, by the definition of $F(a)$ in (4.3), we get

$$
F(0)=X(0)<0
$$

In the case of $X \rightarrow X_{0} a^{-n},(a \rightarrow+0, n>0)$, again by (4.7) and condition (3.54), we have

$$
P_{\mathrm{m}} \rightarrow \frac{(n+1) X_{0}}{\kappa a^{4+n}}, \quad(a \rightarrow+0) .
$$

by $P_{\mathrm{m}}<0$ we find $X_{0}<0$. According to the definition of $F(a)$ in (4.3), we get

$$
F \rightarrow \frac{X_{0}}{a^{n}}<0, \quad(a \rightarrow+0) .
$$

Then we prove (4.8) holds in all cases.

The above theorem implies the following important conclusion,

\section{$a>0$, the evolution of the universe can not reach the initial singularity.}

Now we check $a>0$. For the solution of Friedmann equation (4.3), we have $F(a)=a^{\prime 2} \geq 0$. By (4.8) and the continuity of $F(a)$, the equation $F(a)=0$ certainly have a positive $\operatorname{root} 0<a_{0} \ll \bar{R}$. If $F(a)=0$ has only this positive root $a_{0}$, by $F(a) \in C^{1}$, then $F(a)$ can be expressed as

$$
F(a)=\left(a-a_{0}\right) A(a), \quad\left(A>0, \forall a \geq a_{0}\right)
$$

If $F(a)=0$ has a series of different positive roots $0<a_{0}<a_{1}<a_{2}<\cdots, F(a)$ for the practical universe should be simply connected and then can be expressed as

$$
F(a)=\left(a-a_{0}\right)\left(a_{1}-a\right) B(a), \quad\left(B>0, a_{0} \leq a \leq a_{1}\right)
$$




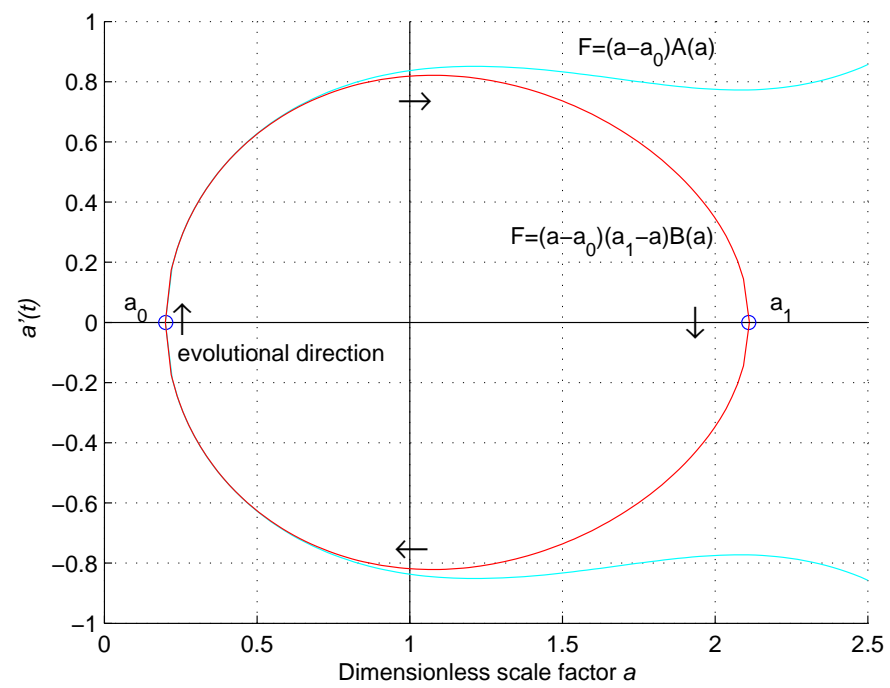

Figure 1: The phase trajectories of Friedmann equation (4.3)

Since Friedmann equation is an equation in average sense, the multiple roots are meaningless in physics. The connected phase trajectories $a \sim a^{\prime}$ of dynamical equation (4.3) with (4.13) or (4.14) are displayed in Fig.1, in which we have set the mean scale $\bar{R}=1$. (4.14) corresponds to the cyclic cosmological model, and (4.13) to the bouncing one. We set the time origin $t=0$ at the turning point $a(0)=a_{0}$. Form Fig:1 we learn clearly the initial singularity is absent, i.e., $a$ can not reaches 0 point.

Substituting (4.13) or (4.14) into (4.5) and letting $a=a_{0}$, in both cases we have

$$
\rho_{\mathrm{m}}\left(a_{0}\right)=\frac{3}{\kappa a_{0}^{2}}\left(K-\frac{1}{3} \Lambda a_{0}^{2}\right)>0 .
$$

Since $\Lambda \geq 0$ in cosmology, by (4.15) we certainly have $K=1$ due to $\rho_{\mathrm{m}}>0$. Then we get another conclusion:

\section{$K=1$, the space of the universe is a closed 3 dimensional sphere $S^{3}$.}

In the cyclic closed case (4.14), we have an estimation of upper bound for the cosmological constant $\Lambda$. Substituting (4.14) into (4.5) and letting $a=a_{1}$, by (3.53) we have

$$
\rho_{\mathrm{m}}\left(a_{1}\right)=\frac{3}{\kappa a_{1}^{2}}\left(1-\frac{1}{3} \Lambda a_{1}^{2}\right)>0 .
$$

Since $\bar{R} a$ forms the main part of mass-energy density at present time, which can be estimated by observational data 21], and $|X(a)| \ll \bar{R} a$ as $a \rightarrow \infty$ can be omitted. For $\Lambda \geq 0$ we have $a_{1} \geq 2 \bar{R}$ and estimation

$$
0 \leq \Lambda<\frac{3}{a_{1}^{2}} \leq \frac{3}{4 \bar{R}^{2}} \sim 10^{-24} \mathrm{ly}^{-2}
$$


So for the cosmological constant $\Lambda$ in a cyclic and closed universe, we get the third conclusion:

$$
0 \leq \Lambda<10^{-24} \mathrm{ly}^{-2}, \text { the cosmological constant is an infinitesimal. }
$$

This estimation is less than the present observational data. This difference can be explained by the potentials $W$ in energy-momentum tensor, which is a fast decaying $\Lambda(a)$ in Friedmann equation. Therefore setting constant $\Lambda=0$ is a good choice in cosmology.

For a bouncing cosmological model, while $a \rightarrow \infty$, the behavior of Friedmann equation (4.3) is controlled by dominant term $\frac{1}{3} \Lambda a^{4}$, and the fast decaying term $X(a) / a \rightarrow 0$ can be omitted. In this case, to be clear, (4.3) can be replaced by the following dimensionless Hamiltonian-like equation

$$
\frac{1}{2} q^{2}+V(q)=H, \quad V(q) \equiv \frac{1}{6} \lambda q^{4}-\frac{1}{54} q(q+6)(q-3)^{2},
$$

in which

$$
q(t)=\frac{a(t)}{\bar{R}}, \quad \lambda=\frac{1}{9}-\Lambda \bar{R}^{2}
$$

For solution of Friedmann equation we have $H \equiv 0$, which is conserved.

In (4.18), $q(t)$ is equivalent to the coordinate of a unit mass, $V(q)$ potential, and $H$ energy. The potential function $V(q)$ and phase trajectories $\left(q^{\prime}, q\right)$ is displayed by Fig.2 and Fig. 3 . The solution

is closed if $\lambda>0 \Leftrightarrow \Lambda<\frac{1}{9} \bar{R}^{-2} \sim 10^{-25} \mathrm{ly}^{-2}$, but bouncing if $\lambda \leq 0 \Leftrightarrow \Lambda \geq \frac{1}{9} \bar{R}^{-2}$. As we can see from Fig, 2 and Fig, $3, \lambda$ or $\Lambda$ has only influence on the behavior of a fully developed universe but no influence on a small universe. On the contrary, the function of $X(a)$ can prevent $a(t)$ from reaching the origin but no influence on a fully developed universe. For closed universe, we have the second root $2 \bar{R} \leq a_{1}<3 \bar{R}$. In contrast $\Lambda<\frac{1}{9} \bar{R}^{-2}$ with (4.17), we find the estimation of $\Lambda$ by criterion $\rho_{\mathrm{m}}>0$ is larger. In bouncing case[25], we have $\left(\frac{d}{d \tau} a\right)^{2} \rightarrow C^{2} a^{2} \Leftrightarrow a \rightarrow C_{0} \cosh (C \tau)$ as $\tau \rightarrow \pm \infty$.

The bouncing model with closed space is inconsistent with the isotropy and homogeneity of the present universe, because the universe should be heavily anisotropy and inhomogeneity before the turning point $t<0$ due to the lack of initial causality among remote parts, and some information should be kept today. From the above analysis we find $\Lambda a^{4}$ is purely a trouble term without any practical purpose.

\section{DISCUSSION AND CONCLUSION}

Since the above derivations are all elementary and reliable, and all concepts have clearly physical meanings, so the conclusions should be quite credible. However, the above conclusions contradict 


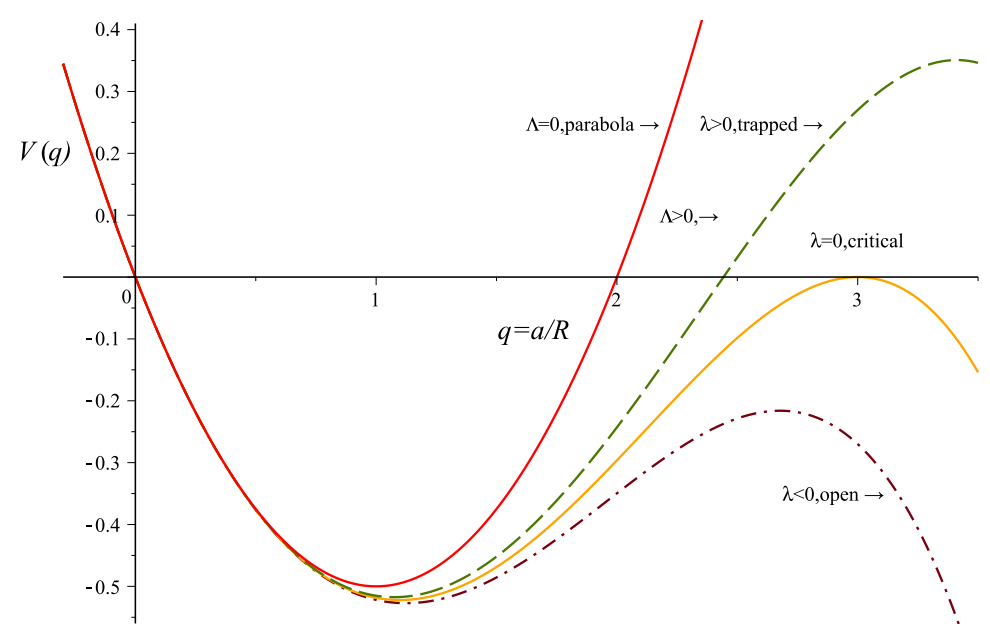

Figure 2: Potential $V(q)=\frac{1}{6} \lambda q^{4}-\frac{1}{54} q(q+6)(q-3)^{2}$ defined in (4.18).

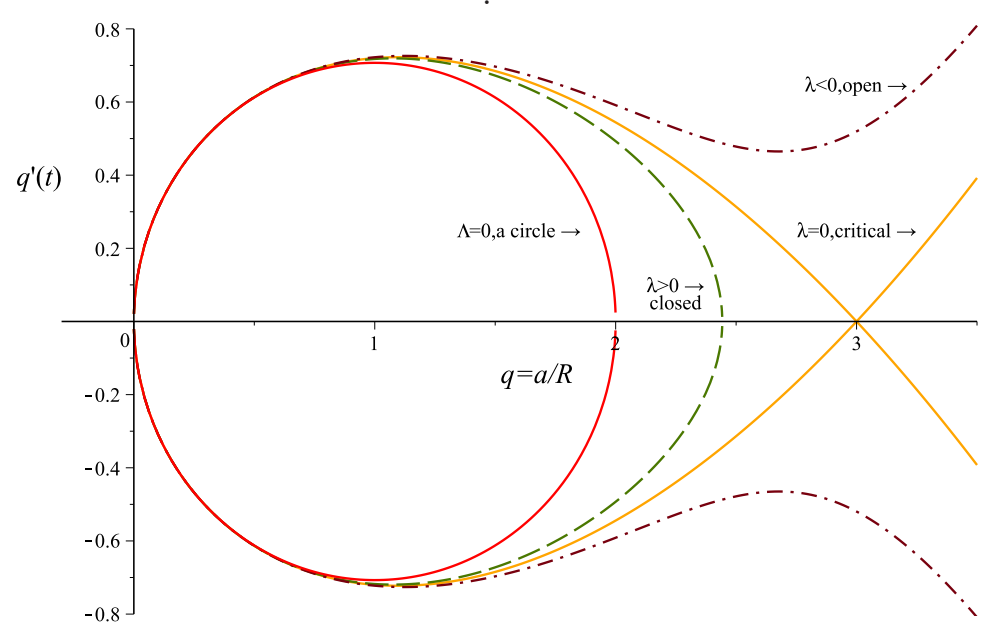

Figure 3: Phase trajectories of $\frac{1}{2} q^{\prime 2}+V(q)=0$ similar to Fig.1. Inside the circle $\Lambda<0$ and outside $\Lambda>0$. In Friedmann equation (4.3), $X(a)<0(a \rightarrow+0)$ removes the Big Bang .

the singularity theorems, this is because some preconditions of these theorems are invalid in physical world[25]. At first, the existence of negative pressure or potential is ignored in the energy condition. Secondly, the closed trapped surface cannot form dynamically, because the EMT manifestly includes the motion of particles, and the center of a star is an unbalance point for particles where particles cannot accumulate and stay statically in heavily curved space. Gravity is a conservative force in which the mechanical energy of the particles is conserved. Considering this compatible factor, we always get singularity-free stars [44]. Besides, we have only unique realistic simultaneous Cauchy surface in the space-time[30, 45], but the derivation of Raychaudhury equation unconsciously assumes and uses the future properties of the space-time and violates this requirement. 
So this equation cannot be generally used for dynamical analysis. One counterexample is enough to disprove a theorem. We have to reappraise the singularity in physics due to this inviolable principle. A realistic singularity in Nature is actually contradictory, lawless and incomprehensible, so the absence of singularity in Nature is a basic principle in physics [22, 32].

In addition to rethinking on the technical issues of standard models and singularity theorems, we should also rethink on our researching methods and academic environment. For example, the closed universe models published in [21, 22] had been completed in 1998 [46], and its researching methods are clearly scientific and strict, but it was repeatedly rejected. The main content of this paper was completed in 2007(see arXiv:0709.2414), and the preliminaries [28, 31, 41, 43, 44] were completed even early, but they have been rejected dozens of times by professional journals, and the reasons of rejection are all inexplicable. Many physicists can only enjoy fantasies or wild ideas such as parallel universes, multidimensional space, modification of gravity, quantum entanglement and so on[24], and it is called 'innovation', but no longer care about causality and logic relation among fundamental concepts. From the review reports we find that many referees cannot actually understand the above derivation, for example, the introduction of intermediate function $X(a)$ in the (4.3) for convenience of analysis is rated as lacking creativity. This situation greatly hinders the normal academic communication and scientific progress. The fundamental physics has stagnated for nearly a hundred years, and the unhealthy academic environment should bear important responsibility.

To sum up, by studying the properties of the EMT and EOS of various physical fields, and qualitatively analyzing the dynamical behavior of the general Friedmann equation and logical relations between parameters, we get some definite constraints on $(K, \Lambda)$. We find that only the cyclic and closed cosmological model with a tiny or vanishing $\Lambda$ is natural and reasonable in physics. The other cases include nonphysical effects or logical contradictions. Such constraints will be helpful for the research of some other issues in cosmology. In some sense, we restored Heraclitus' ancient faith: "The world, an entity out of everything, was created by neither gods nor men, but was, is and will be eternally living fire, regularly becoming ignited and regularly becoming extinguished."

[1] A. G. Riess et al. (Supernova Search Team), Astron. J. 116: 1009 (1998), astro-ph/9805201.

[2] S. Perlmutter et al. (Supernova Cosmology Project), Astrophys. J. 517: 565(1999).

[3] N. Spergel et al. (WMAP), Astrophys. J. Suppl. 148: 175(2003), astro-ph/0302209. 
[4] M. Tegmark et al. (SDSS), Phys. Rev. D69: 103501(2004), astro-ph/0310723.

[5] J. Dunkley et al. The Atacama Cosmology Telescope: Cosmological Parameters from the 2008 Power Spectra, Astrophys. J. Vol.739, No.1 (2011), arXiv:1009.0866

[6] J. L. Sievers et al. The Atacama Cosmology Telescope: Cosmological parameters from three seasons of data, J. Cosm. Astro. Phys., 2013 (10): 60(2013), arXiv:1301.0824

[7] V. Sahni, The cosmological constant problem and quintessence, Class. Quant. Grav., 19: 34353448(2002), astro-ph/0202076

[8] P. J. E. Peebles, B. Ratra, The Cosmological Constant and Dark Energy, Rev. Mod. Phys. 75: 559606(2003), astro-ph/0207347

[9] M. S. Turner, D. Huterer, Cosmic Acceleration, Dark Energy and Fundamental Physics, J.Phys. Soc. Jap.76: 111015(2007), arXiv:0706.2186

[10] M. Ishak, Remarks on the formulation of the cosmological constant/dark energy problems, Found.Phys.37: 1470-1498(2007), astro-ph/0504416

[11] M. Szydlowski, A. Kurek, A. Krawiec, Top ten accelerating cosmological models, Phys. Lett. B642: 171-178(2006), astro-ph/0604327

[12] Marek Szydlowski, Cosmological zoo - accelerating models with dark energy, JCAP0709: 007(2007)

[13] E. J. Copeland, M. Sami, S. Tsujikawa, Dynamics of dark energy, IJMPD 15: 1753-1936(2006)

[14] E. V. Linder, Theory Challenges of the Accelerating Universe, J. Phys. A40: 6697(2007)

[15] Ph. Bull, Y. Akrami, et al., Beyond $\Lambda$ CDM: Problems, solutions, and the road ahead, Physics of the Dark Universe 12: 56-99(2016), arXiv:1512.05356

[16] P. J. Steinhardt, N.Turok, A Cyclic Model of the Universe, Science 296: 1436(2002), hep-th/0111030

[17] P. J. Steinhardt, N.Turok, The Cyclic Model Simplified, New Astron. Rev. 49: 43-57(2005)

[18] J. Khoury, P. J. Steinhardt, N.Turok, Designing Cyclic Universe Models, Phys. Rev. Lett. 92: 031302(2004), hep-th/0307132

[19] J. D. Barrow, M. P. Dabrowski, Oscillating universes, Mon. Not. Astron. Soc. 275: 850-862(1995).

[20] J. D. Barrow, D. Kimberly, J. Magueijo, Bouncing Universes with Varying Constants, Class. Quant. Grav., 21 (18): 57-61(2013), astro-ph/0406369

[21] Y. Q. Gu, A Cosmological Model with Dark Spinor Source, Int. J. Mod. Phys. A22: 4667-4678(2007), gr-qc/0610147.

[22] Y. Q. Gu, Clifford Algebra, Lorentz Transformation and Unified Field Theory, Adv. Appl. Cliff. Alg. 28(2):37 (2018). https://doi.org/10.1007/s00006-018-0852-0

[23] M. Madsen and G. Ellis, Evolution of in in ationary universes, Mon. Not. Roy. Astron. Soc. 234 (1988) 67.

[24] George F.R. Ellis, Emma Platts, David Sloan, Amanda Weltman, Current observations with a decaying cosmological constant allow for chaotic cyclic cosmology, JCAP 04 (2016) 026

[25] M. Novello, S.E.P. Bergliaffa, Bouncing cosmologies, Physics Reports 463 (2008) 127-213.

[26] Di Valentino, E., Melchiorri, A., Silk, J. Planck evidence for a closed Universe and a possible crisis for 
cosmology. Nat. Astron. (2019) doi:10.1038/s41550-019-0906-9

[27] J. M. Nester, Special orthonormal frames, J. Math. Phys. 33, 910 (1992).

[28] Y. Q. Gu, The Vierbein Formalism and Energy-Momentum Tensor of Spinors, gr-qc/0612106

[29] Y. Q. Gu, Space-Time Geometry and Some Applications of Clifford Algebra in Physics. Adv. Appl. Clifford Algebras, 2018, 28(4):79.

[30] Y. Q. Gu, Natural Coordinate System in Curved Space-time, J. Geom. Symmetry Phys. 47 (2018) 51“C62, arXiv:gr-qc/0612176, doi: 10.7546/jgsp-47-2018-51-62

[31] Y. Q. Gu, The Simplification of Spinor Connection and Classical Approximation, arXiv:gr-qc/0610001

[32] Y. Q. Gu, Local Lorentz Transformation and Mass-Energy Relation of Spinor, Physics Essays Vol. 31: 1-6(2018). arXiv:hep-th/0701030

[33] M. Ozer and M. O. Taha, A Possible Solution to the Main Cosmological Problems, Phys. Lett. B 171, 363-365 (1986).

[34] M. Ozer and M.O. Taha, A Model of the Universe Free of Cosmological Problems, Nuclear Phys. B 287, 776-796 (1987).

[35] W. Chen, Y. Wu, Implications of a Cosmological Constant Varying as $R^{-2}$, Phys. Rev., 41, 695-698 (1990).

[36] M. Berman, Cosmological Models with a Variable Cosmological Term, Phys. Rev. D 43, 1075-1078 (1991).

[37] A.S. Al-Rawaf and M.O. Taha, Cosmology of General Relativity without Energy-Momentum Conservation, Gen. Rel. Grav. 28, 935-952 (1996).

[38] A.S. Al-Rawaf, A Cosmological Model with a Generalized Cosmological Constant, Mod. Phys. Lett. A 13, 429-432 (1998).

[39] J.M. Overduin and F.I. Cooperstock, Evolution of the Scale Factor with a Variable Cosmological Term, Phys. Rev. D 58, 043506:1-23 (1998).

[40] A.I. Arbab, Cosmic Acceleration with a Positive Cosmological Constant, Class. Quant. Grav. 28, 93-99 (2003).

[41] Y. Q. Gu, Functions of State for spinor gas in General Relativity, OALib. J. 4, e3953 (2017). arXiv:0711.1243

[42] J. D. Barrow, Finite Action Principle Revisited, Phys. Rev. D 101, 023527 (2020)

[43] Y. Q. Gu, Nonlinear Spinors as the Candidate of Dark Matter, OALib. J. 4, e3954(2017), arXiv:0806.4649

[44] Y. Q. Gu, Structure of the Star with Ideal Gases, arXiv:0712.0219

[45] Y. Q. Gu, Some Subtle Concepts in Fundamental Physics, Physics Essays 30: 356-363(2017), arXiv:0901.0309

[46] Y. Q. Gu, The Electromagnetic Potential Among Nonrelativistic Electrons, Adv. Appl. Cliff. Alg. 9(1), 61-79(1999) 